\title{
Precuneus atrophy in early-onset Alzheimer's disease: a morphometric structural MRI study
}

\author{
Giorgos Karas • Philip Scheltens • Serge Rombouts • \\ Ronald van Schijndel • Martin Klein • Bethany Jones • \\ Wiesje van der Flier • Hugo Vrenken • Frederik Barkhof
}

Received: 3 January 2007 / Accepted: 6 June 2007 / Published online: 23 October 2007

(C) Springer-Verlag 2007

\begin{abstract}
Introduction Alzheimer's disease (AD) usually first presents in elderly patients, but may also develop at an earlier age. Patients with an early age at onset tend to present with complaints other than memory impairment, such as visuospatial problems or apraxia, which may reflect a different distribution of cortical involvement. In this study we set out to investigate whether age at onset in patients with $\mathrm{AD}$ determines the pattern of atrophy on cerebral MRI scans.
\end{abstract}

\section{G. Karas $(\bowtie) \cdot$ F. Barkhof}

Department of Diagnostic Radiology,

Vrije Universiteit Medical Centre,

De Boelelaan 1117,

1081 HV Amsterdam, The Netherlands

e-mail: GB.Karas@vumc.nl

R. van Schijndel $\cdot$ H. Vrenken $\cdot$ F. Barkhof

Image Analysis Center,

Vrije Universiteit Medical Center,

Amsterdam, The Netherlands

G. Karas $\cdot$ P. Scheltens $\cdot$ S. Rombouts $\cdot$ B. Jones $\cdot$

W. van der Flier $\cdot$ F. Barkhof

Alzheimer Center, Vrije Universiteit Medical Center,

Amsterdam, The Netherlands

S. Rombouts $\cdot$ R. van Schijndel

Department of Clinical Physics and Informatics,

Vrije Universiteit Medical Center,

Amsterdam, The Netherlands

P. Scheltens $\cdot$ B. Jones

Department of Clinical Neurology,

Vrije Universiteit Medical Center,

Amsterdam, The Netherlands

\section{Klein}

Department of Medical Psychology,

Vrije Universiteit Medical Center,

Amsterdam, The Netherlands
Methods We examined 55 patients with $\mathrm{AD}$ over a wide age range and analyzed their 3-D T1-weighted structural MRI scans in standard space using voxel-based morphometry (VBM). Regression analysis was performed to estimate loss of grey matter as a function of age, corrected for minimental state examination (MMSE) scores and sex.

Results The VBM analyses identified multiple areas (including the temporal and parietal lobes), showing more atrophy with advancing age. By contrast, a younger age at onset was found to be associated with lower grey matter density in the precuneus. Regionalized volumetric analysis of this region confirmed the existence of disproportionate atrophy in the precuneus in patients with early-onset AD. Application of a multivariate model with precuneus grey matter density as input, showed that precuneal and hippocampal atrophy are independent from each other. Additionally, we found that a smaller precuneus is associated with impaired visuospatial functioning.

Conclusion Our findings support the notion that age at onset modulates the distribution of cortical involvement, and that disproportionate precuneus atrophy is more prominent in patients with a younger age of onset.

Keywords Early-onset Alzheimer's disease . Voxel-based morphometry Precuneus

\section{Introduction}

Alzheimer's disease (AD) is the most common form of dementia, with a typical age at onset beyond 75 years of age. In elderly patients, the clinical hallmark of the disease is progressive memory impairment, followed by global cognitive decline. Accordingly, atrophy of the medial temporal lobe (MTA), including the hippocampus, is one 
of the most important markers on magnetic resonance imaging (MRI). However, AD may also develop at an earlier age, and these patients tend to present with complaints other than memory impairment, such as visuospatial problems or apraxia $[1,2]$, probably reflecting a different pattern of cortical involvement.

Therefore, regional cortical atrophy patterns may differ between patients with early-onset and those with late-onset $\mathrm{AD}$ (EAD and LAD, respectively). So far, most MRI studies in $\mathrm{AD}$ have focused on elderly patients. Various MRI studies have suggested more posterior atrophy in patients with EAD [3, 4]. Comparable areas in the brain have been identified with PET/SPECT studies [5-7]. One of the areas incriminated is the precuneus.

The precuneus is located in the medial aspect of the posterior parietal lobe, and its borders are the parietooccipital sulcus posteriorly and the marginal ramus (pars marginalis) of the cingular sulcus anteriorly. The precuneus is best appreciated on a midsagittal section. It lies immediately anterior to the cuneus and posterior to the posterior cingulate cortex (PCC) and retrosplenial cortex, while more superiorly it is separated from the primary sensorimotor cortex by the marginal ramus of the cingular sulcus. Cytoarchitecturally, the precuneus corresponds to Brodmann area 7, an area in close relationship to Brodmann areas 31 (PCC) and 23 (PCC proper). Cortical and subcortical connectivity of the precuneus is extensive [8]: medial and lateral parietal cortex, frontal cortex, superior temporal sulcus, thalamus, striatum, claustrum and brainstem. As an additional analysis we attempted to explore a possible association between precuneus atrophy and specific neuropsychological deficits in patients with EAD [9-11].

In the present study, we aimed to assess atrophy according to age at onset in a sample of patients with AD. We hypothesized that elderly patients with $\mathrm{AD}$ would show mainly MTA, while in younger patients with AD atrophy might be observed outside the temporal lobe, including the parietal lobe and precuneus.

\section{Patients and methods}

Inclusion of patients

Consecutive patients with $\mathrm{AD}$ were recruited at the secondary/tertiary referral Alzheimer Center at our institute. All patients underwent a standardized work-up that involved history taking, physical and neurological examination, blood tests (erythrocyte sedimentation rate, haemoglobin, white cell count, serum electrolytes, glucose, creatinine, liver function tests, thyroid stimulation hormone and free thyroid hormone, vitamin B1 and B6 levels, and syphilis serology), Mini Mental State Examination (MMSE), comprehensive neuropsychological examination when indicated, and MRI of the brain. A diagnosis of probable AD was based upon the NINCDS-ADRDA criteria [12]. Only those AD patients whose diagnosis remained unchanged after a minimum follow-up of 1 year were included in this study. In the absence of neuropathological confirmation, this approach was used in order to improve the accuracy of the diagnosis [13]. All patients provided written informed consent.

Image acquisition and processing

MR imaging was performed on a 1.0-T Magnetom Impact Expert system (Siemens, Erlangen, Germany). For the anatomical high-resolution scans, whole-brain T1-weighted 3-D MPRAGE (magnetization-prepared rapid-acquisition gradient echo) volumes were acquired in the coronal plane (TR $15 \mathrm{~ms}$, TE $7 \mathrm{~ms}$, TI $300 \mathrm{~ms}$, number of excitations 1; flip angle $15^{\circ}$ ). Voxel sizes were $1 \times 1 \times 1.5 \mathrm{~mm}$.

MTA on the coronal MPRAGE scans was visually scored according to a well-established scale [14]. This scale is based on a visual estimation of the medial temporal lobe, encompassing the hippocampus proper, dentate gyrus, subiculum, parahippocampal gyrus and the volume of the surrounding cerebrospinal fluid (CSF) spaces, especially the temporal horn of the lateral ventricle and the choroid fissure, bilaterally. The MTA score ranges from 0 (no atrophy) to 4 (severe atrophy). Visual scores from left and right were averaged.

Global grey matter (GM) volume was estimated with a cross-sectional atrophy estimation method (SIENAX: structural image evaluation, including normalization, of atrophy [15]). Briefly, scans were registered to standard space, the skull and brain were extracted and the GM was segmented based on signal intensity, atlas information and a voxelconnectivity algorithm. Subsequently, GM global volumes were corrected for scaling and scanner errors by using the skull as scaling parameter, and partial volume effects were incorporated into to the model. The resulting GM global volumes were then expressed as cubic centimetres.

Localized GM differences were assessed with voxelbased morphometry (VBM) [16-18]. MRI scans were registered using the following methods. Initially the MRI scans were brought to standard space by utilizing the registration algorithm of statistical parametric mapping (SPM) (affine 12 degrees of freedom registration with least-squares minimization as the function). Subsequently the affine registration was refined by a more advanced template-less approach by spreading registration bias among the whole group-transformation matrix averaging by projection on a manifold [19]. The latter method incorporates a 12 degrees of freedom affine approach with least-squares minimization as the cost function.. Spreading the registration error among group members creates a 
template with more clearly defined gyri than ordinary registration (Fig. 1). The second step was to account for finer anatomical variability by utilizing the low-frequency basis function nonlinear approach of SPM with $7 \times 8 \times 7$ basis functions and medium regularization (the metric used to constrain the warping effect of the algorithm). Although there are high-dimensional nonlinear registration algorithms with millions of degrees of freedom (voxel-compression mapping, for example [20]), the use of such an algorithm would have moved us away form VBM towards tensorbased morphometry. VBM brings homologous anatomical regions into close proximity and performs well compared to other higher-dimensional registration algorithms [21]. Subsequently, scans were segmented into GM, white matter (WM) and CSF, based on an atlas-driven expectation maximization-maximum likelihood (EM-ML) segmentation algorithm, which also included bias field inhomogeneity correction and produced statistical probability anatomical maps (SPAMs). SPAM values range from $0 \%$ to $100 \%$ probability for a voxel belonging to a tissue class (GM, WM, or CSF). Taking into account that scanner field inhomogeneities and tissue signal intensity profiles might differ between scanners we sought expert help from a neuroradiologist in order to define the threshold of SPAMs, to minimize the effects of over- and undersegmentation of GM; the optimum for our images was found to be at $79 \%$ threshold for the SPAMs. The resulting GM maps were smoothed with a Gaussian kernel of $12 \mathrm{~mm}$ (full-width at half-maximum), which is a suitable setting for detecting atrophy [22].

VBM data were then analyzed with SPM. In order to measure the effect of age on GM a "single-subject covariatesonly" model was built in SPM, with age as the single covariate and sex and MMSE as nuisance variables. The output of this type of analysis produces two maps: a positive VBM map (indicating more atrophy in young patients) and a negative

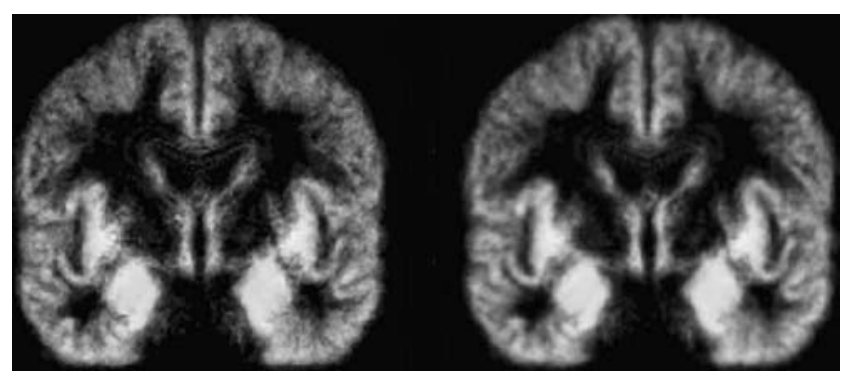

Fig. 1 Effect of registration error spreading among all scans in the group. The method used was transformation matrix averaging by projection onto a semi-Riemannian manifold [19]. On the left image scans are mapped to standard space and subsequently averaged to create a template. On the right image scans are also mapped to standard space but subsequently registration error was spread among all scans. Notice that the resulting template on the right image shows clearer delineation of gyri
VBM map (indicating more atrophy in older patients). The negative map, on which areas of cerebral atrophy correlate with age, entails the mixed effects of disease, disease duration and normal aging. The positive map shows areas that are more atrophic in younger patients or less atrophic in older patients; this map shows only disease differences between the two groups since this map has the opposite direction of aging. Subsequently, the positive VBM map was regionalized and the normalized volume per scan was calculated (termed regionalized volume, $\mathrm{RV}$ ).

\section{Statistical analysis of variables}

Either parametric (Pearson) or nonparametric (Spearman) correlation coefficients were calculated between age and the following variables: SIENAX global GM volume, MTA score, RV and sex.

\section{Technical issues}

VBM analysis was performed with SPM2, running under Matlab 6.5 (Mathworks, Natick, Mass.). Special image processing steps were implemented in IDL 6.1 (Research Systems, Boulder, Colo.). Cluster extraction was done with the SPM plug-in 'mars bar' [23]. Conversion of the Montreal Neurological Institute (MNI) coordinates to Talairach coordinates was made possible with the 'mni2tal.m' script in Matlab. Special Matlab, IDL and UNIX shell scripts were used to batch process the analysis. All extra scripts and source code are freely available upon request from the author. SPSS 13 was used for conventional statistical analysis.

\section{Post-hoc neuropsychological analysis}

In patients referred to the memory clinic (28 of 32 with LAD, 11 of 19 with EAD), a battery of standard tests was employed to assess neuropsychological functioning (Table 2). The time required to complete the battery was approximately 90 minutes. Screening included tests of processing speed (WAIS Digit Symbol Substitution [24], Trail Making A [25]), attention (Digit Span [24]), episodic memory (Visual Association Test, a brief learning task based on imagery mnemonics that detects with high specificity AD patients a year before the clinical diagnosis [26]), semantic memory (categorical word fluency: 1 minute animals, 1 minute insects [27] and WAIS Information [24]), executive functioning (Trail Making B [25] and meander drawing [28]), and a test of visuospatial functioning [29]. Objective cognitive impairment was defined as a score of more than 1.5 standard deviations below the mean of age-, education-, and gender-matched controls. For a subgroup of EAD patients neuropsychological testing had already been performed elsewhere, and the data were not available for review. 
In order to investigate a possible association of precuneus atrophy with visuospatial functioning, precuneus volume was compared to a metric of visuospatial functioning (drawing task). Since missing data in this analysis might have constituted an important confounder, we also compared precuneus volume between the patients with missing data and the patients with available data. There was no difference between the two groups ( $t$-test, $P=0.58)$; therefore bias had not been introduced.

\section{Results}

A total of 55 patients with AD were included in this study; four patients were excluded from the analysis due to MR imaging artefacts or grossly enlarged lateral ventricles which caused the image processing pipeline to fail. The average age of the $\mathrm{AD}$ group was 69 years (SD 8.5) and with a wide range of 51-82 years, encompassing both younger and older AD patients. MMSE scores had an average of 20 (SD 6, range 4-28). Gender distribution was balanced with 29 women and 22 men. Age was positively correlated with MMSE (Spearman's rho $=0.43$ at $P<0.005$ ), indicating that younger patients were more severely affected. Age was also correlated with MTA (Spearman's rho $=0.27$ at $P<0.05$ ), indicating that older subjects had more hippocampal atrophy. Precuneus regionalized volume was lower in younger patients (Pearson's $r=0.4$ at $P<0.004$ ). There was a trend for a global GM volume decline with age but this was not statistically significant (Pearson's $r=-0.22$ at $P=0.15$ ).

The VBM correlation analyses (corrected for clusterextent multiple comparisons at $P<0.05$ and corrected for MMSE and sex) revealed multiple areas of advancing atrophy as a function of advancing age: older patients had more atrophy in the left superior and inferior temporal gyrus, the pre- and postcentral gyrus, the left superior frontal gyrus and the left thalamus (Table 1). The reversed correlation analysis (positive VBM map) demonstrated more atrophy of the precuneus in younger patients (Fig. 2). In order to ensure that the findings were not biased by our segmentation threshold, we also analyzed nonthresholded maps of GM, and the precuneus still remained more atrophic in the

Table 1 Coordinates of age-correlated GM volume regions corrected for multiple comparisons at $P=0.05$ for cluster extent. Due to debate about conversion from MNI to Talairach space, both coordinates are listed [49]

\begin{tabular}{|c|c|c|c|c|c|c|c|}
\hline Comparison & $\begin{array}{l}\text { Coordinate } \\
\text { MNI }\end{array}$ & $\begin{array}{l}\text { Coordinate } \\
\text { Talairach }\end{array}$ & $\begin{array}{l}\mathrm{T} \\
\text { value }\end{array}$ & $\begin{array}{l}\text { Extent } \\
(\mathrm{K})\end{array}$ & $\begin{array}{l}P \text { corrected } \\
\text { cluster level }\end{array}$ & Location & Brodmann \\
\hline \multirow{6}{*}{$\begin{array}{l}\text { Age positive } \\
\text { (younger } \mathrm{AD} \text { ) }\end{array}$} & $-1-6532$ & $-1-6133$ & 5.6 & \multirow[t]{2}{*}{11,819} & \multirow[t]{2}{*}{$<0.001$} & Left precuneus & 7 \\
\hline & $6-5234$ & $6-4934$ & 4.3 & & & Left precuneus & 31 \\
\hline & $-29-525$ & $-29-423$ & 4.6 & \multirow[t]{2}{*}{3,647} & \multirow[t]{2}{*}{$<0.001$} & Left insula & \multirow[t]{2}{*}{13} \\
\hline & $-31-38$ & $-31-37$ & 4.1 & & & Left claustrum & \\
\hline & $38-577$ & $38-559$ & 4.1 & \multirow[t]{2}{*}{1,174} & \multirow[t]{2}{*}{$=0.001$} & Right superior temporal gyrus & 22 \\
\hline & $28-6116$ & $28-5818$ & 3.5 & & & Right posterior cingulate & 31 \\
\hline \multirow{3}{*}{$\begin{array}{l}\text { Age positive (corrected } \\
\text { for SEX + MMSE) }\end{array}$} & $1-6633$ & $1-6234$ & 4.6 & \multirow[t]{3}{*}{5,875} & \multirow[t]{3}{*}{$<0.001$} & Right precuneus & 7 \\
\hline & $7-4934$ & $7-4634$ & 3.9 & & & Right precuneus & 31 \\
\hline & $5-3840$ & $5-3539$ & 3.4 & & & Right posterior cingulate gyrus & 31 \\
\hline \multirow[t]{12}{*}{ Age negative (older AD) } & $-1362-12$ & $-1360-13$ & 4.85 & 1,892 & 0.002 & Left superior frontal gyrus & 11 \\
\hline & $-169-23$ & $-168-20$ & 4.75 & \multirow[t]{3}{*}{10,748} & \multirow[t]{3}{*}{0.000} & Left inferior frontal gyrus & 47 \\
\hline & $-165-45$ & $-163-38$ & 4.55 & & & Left uncus & 38 \\
\hline & $-275-38$ & $-273-32$ & 4.55 & & & Left uncus & 36 \\
\hline & $43-1431$ & $43-1229$ & 4.54 & 1,181 & 0.024 & Right precentral gyrus & \multirow[t]{3}{*}{6} \\
\hline & $-4-210$ & $-4-19$ & 4.5 & \multirow[t]{2}{*}{2,154} & \multirow[t]{2}{*}{0.001} & Left thalamus & \\
\hline & 715 & 715 & 3.76 & & & Right caudate head & \\
\hline & $-45-1435$ & $-45-1233$ & 4.31 & \multirow[t]{2}{*}{1,795} & \multirow[t]{2}{*}{0.002} & Left precentral gyrus & 6 \\
\hline & $-50-1448$ & $-50-1145$ & 4.07 & & & Left postcentral gyrus & 3 \\
\hline & $-5319-12$ & $-52 \quad 18-11$ & 4.06 & \multirow[t]{3}{*}{4,340} & \multirow[t]{3}{*}{0.000} & Left superior temporal gyrus & 38 \\
\hline & $-4530-10$ & $-4529-10$ & 3.99 & & & Left inferior frontal gyrus & 47 \\
\hline & $-3441-10$ & $-3439-10$ & 3.5 & & & Left middle frontal gyrus & 11 \\
\hline \multirow{7}{*}{$\begin{array}{l}\text { Age negative (corrected } \\
\text { for SEX }+ \text { MMSE) }\end{array}$} & $-5018-8$ & $-5017-8$ & 5.01 & \multirow[t]{2}{*}{16,441} & \multirow[t]{2}{*}{0.000} & Left superior temporal gyrus & 38 \\
\hline & $-52-5-35$ & $-51-6-29$ & 4.59 & & & Left inferior temporal gyrus & 20 \\
\hline & $-51-1348$ & $-50-1045$ & 4.5 & \multirow[t]{2}{*}{1,748} & \multirow[t]{2}{*}{0.003} & Left precentral gyrus & 4 \\
\hline & $-46-1337$ & $-46-1135$ & 3.76 & & & Left precentral gyrus & 6 \\
\hline & $-1760-12$ & $-1758-13$ & 4.49 & 1,367 & 0.011 & Left superior frontal gyrus & \\
\hline & $-5-17$ & $-5-16$ & 4.43 & \multirow[t]{2}{*}{2,076} & \multirow[t]{2}{*}{0.001} & Left thalamus & \\
\hline & 716 & 715 & 3.43 & & & Right caudate head & \\
\hline
\end{tabular}


Fig. 2 Location of the VBMcluster showing lower GM probability in younger patients. The maximum value of atrophy corresponds to the precuneus. The bottom left image identifies the boundaries of the precuneus or quadrilateral gyrus, bounded anteriorly by the marginal ramus of the sulcus cingularis and posteriorly by the parietooccipital sulcus. The bottom right image is a stereotactic brain in coronal section with the precuneus marked by asterisks (medially); modified from reference [48]
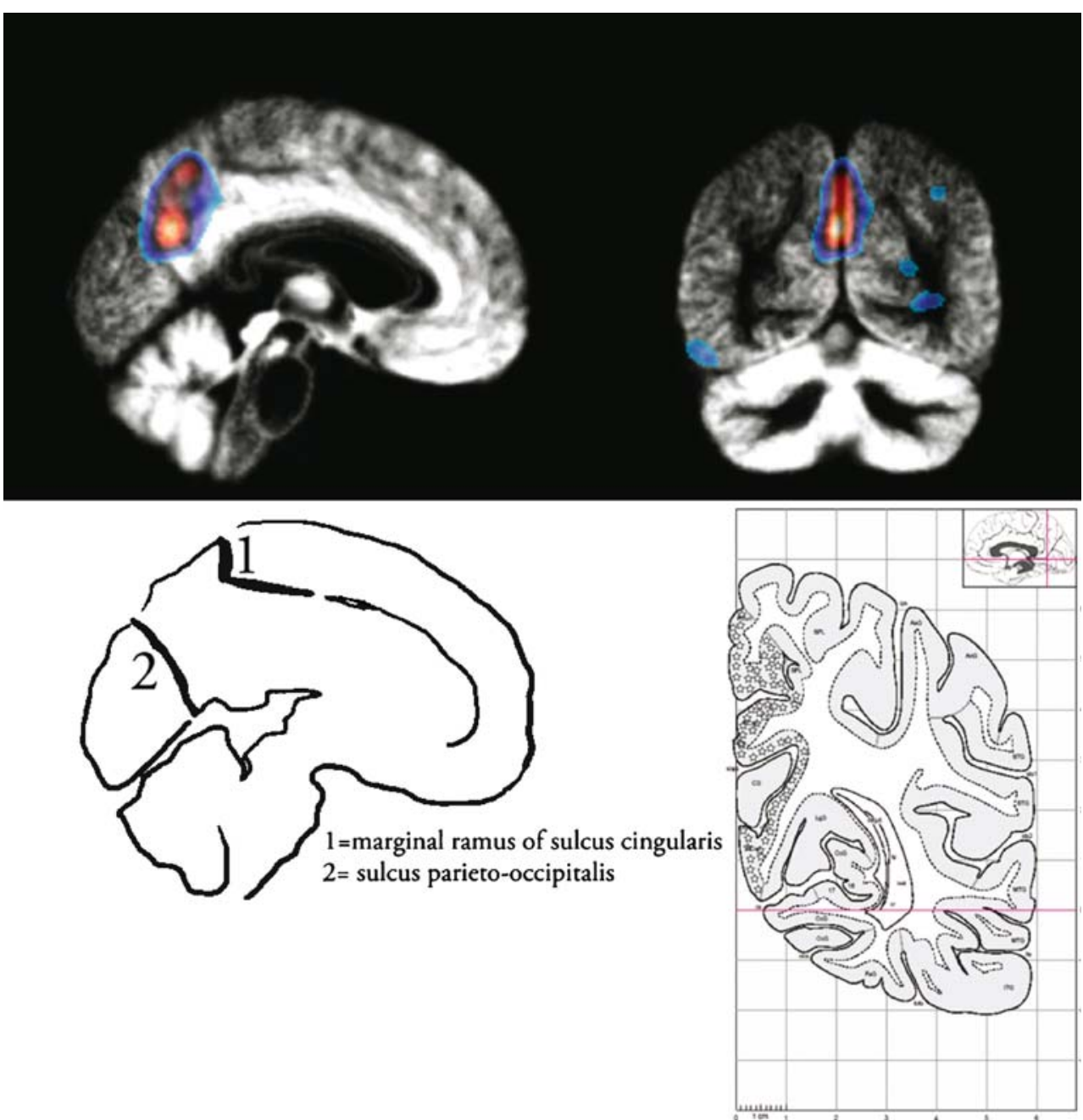

younger patients, albeit at a somewhat lower level of statistical significance $(\operatorname{Tmax}=4.6)$.

The precuneus cluster for the positive VBM map was used to calculate a regionalized average GM volume for this structure (scaled for structure size and plotted against age in Fig. 3). This analysis confirmed a positive correlation between age and the regionalized precuneus volume, indicating that younger AD patients had smaller precunei. The disproportionate atrophy in this area is easily appreciable on the original images, as illustrated in Fig. 4.

SPM is a mass-univariate approach. In order to estimate the relationship between precuneus atrophy and other brain areas, especially the hippocampus, we applied a simple multivariate model in our patients. The regionalized GM volume of the precuneus in each subject was entered into a new model as a single covariate (Fig. 5). The resulting SPM statistical map showed, as expected, a high correlation with the precuneus itself as well as diffuse cortical atrophy bilaterally, with the highest correlations biparietally. There was no association with hippocampal atrophy, even at a lower statistical threshold, effectively protecting our findings from type II error. This indicates that precuneus atrophy occurs completely independently of hippocampal atrophy.

For the sake of completeness and to facilitate comparison with earlier studies, we additionally performed a dichotomous analysis of EAD and LAD patients at an arbitrary cut-off of 65 years of age as used in earlier studies $[3,4]$, using a two-sample $t$-test. Dichotomizing our group produced 35 patients with LAD and 20 patients with EAD (two LAD and two EAD patients were eventually excluded due to image pipeline failure as described above) with average ages of 74.7 years (SD 4.2, range 66-82 years) and 59.5 years (SD 4.3, 51-65 years) and MMSE scores of 21.5 $(5.4,4-28)$ and $16.2(5.6,8-24)$, respectively. The EAD group was characterized by lower MMSE scores $(P<$ $0.002)$, consistent with the correlation analysis. MTA was not significantly different between the two groups $(P=0.3)$, while there was a significant difference in global GM volume between EAD and LAD patients of $6.7 \%(P<0.04)$, with EAD patients having an average GM volume of 
Fig. 3 Scatter plot of precuneus GM volume (corrected for size) versus age

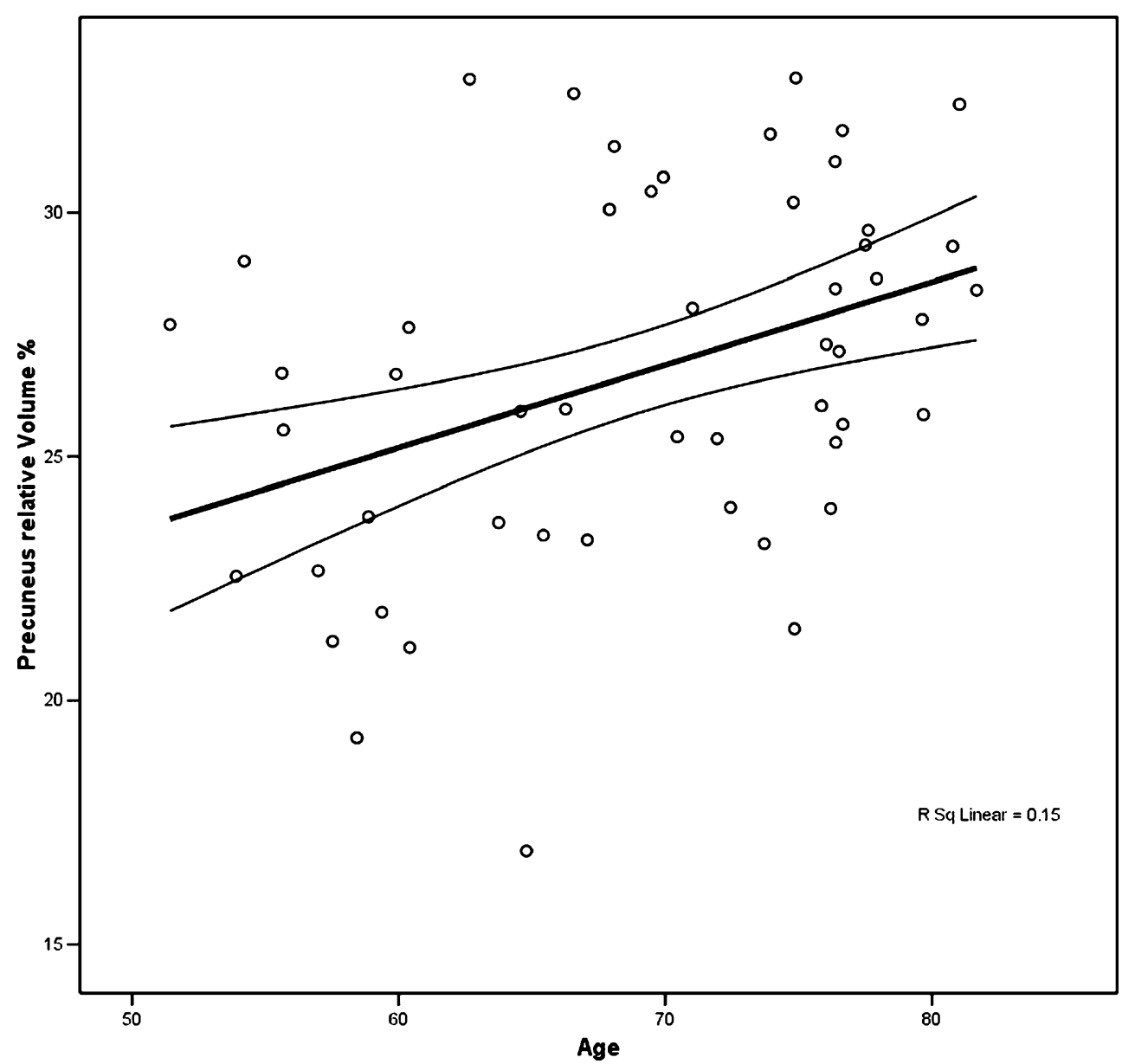

$554 \mathrm{~cm}^{3}$ (SD 60) and LAD patients having an average GM volume of $517 \mathrm{~cm}^{3}$ (SD 58). The dichotomous VBM analysis comparing EAD and LAD patients (with correction for MMSE and sex, and multiple comparison's correction at $P=0.05$ ) was in agreement with the VBM correlation analysis in showing that LAD patients had more atrophy of the left inferior temporal gyrus, left uncus and left superior temporal gyrus, while EAD patients had atrophy of the precuneus (left and right). The left superior frontal gyrus and left thalamus were the only other locations that the dichotomous analysis indicated to be more atrophic in EAD patients.

The subgroup of EAD patients was characterized by a more extensive deterioration in processing speed and attention functioning than LAD patients (Table 2). Analysis of covariance demonstrated that EAD patients performed
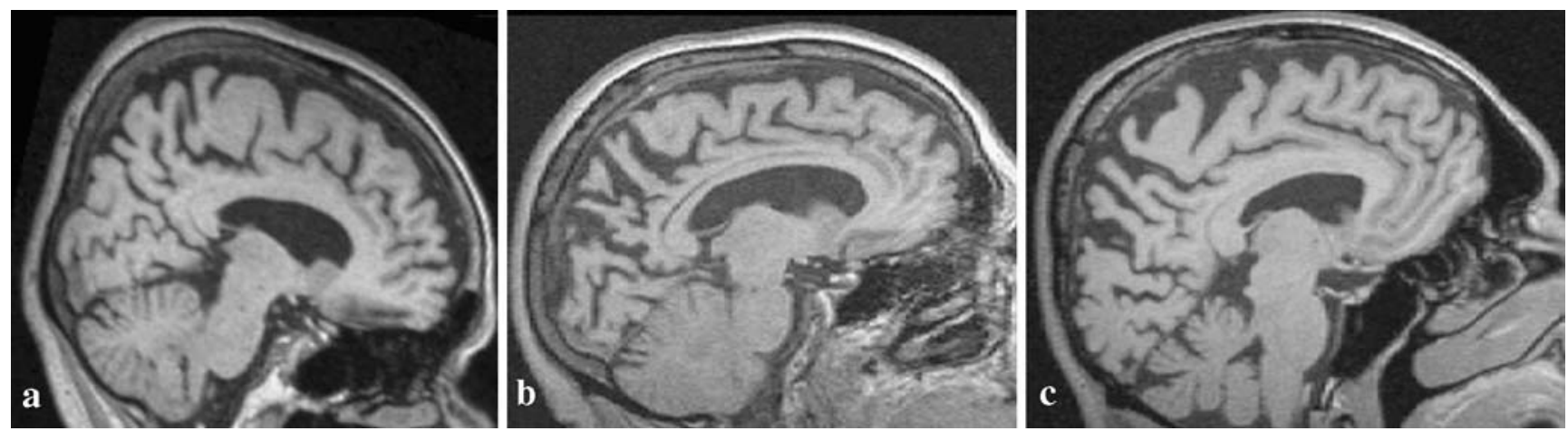

Fig. 4 Three examples of precuneus atrophy best appreciated on multiplanar reformatted images in the sagittal plane. In an atrophic precuneus there is widening of the marginal ramus of the cingulate sulcus and widening of the parietooccipital sulcus (a normal precuneus, $\mathbf{b}$ and $\mathbf{c}$ atrophic precuneus) 


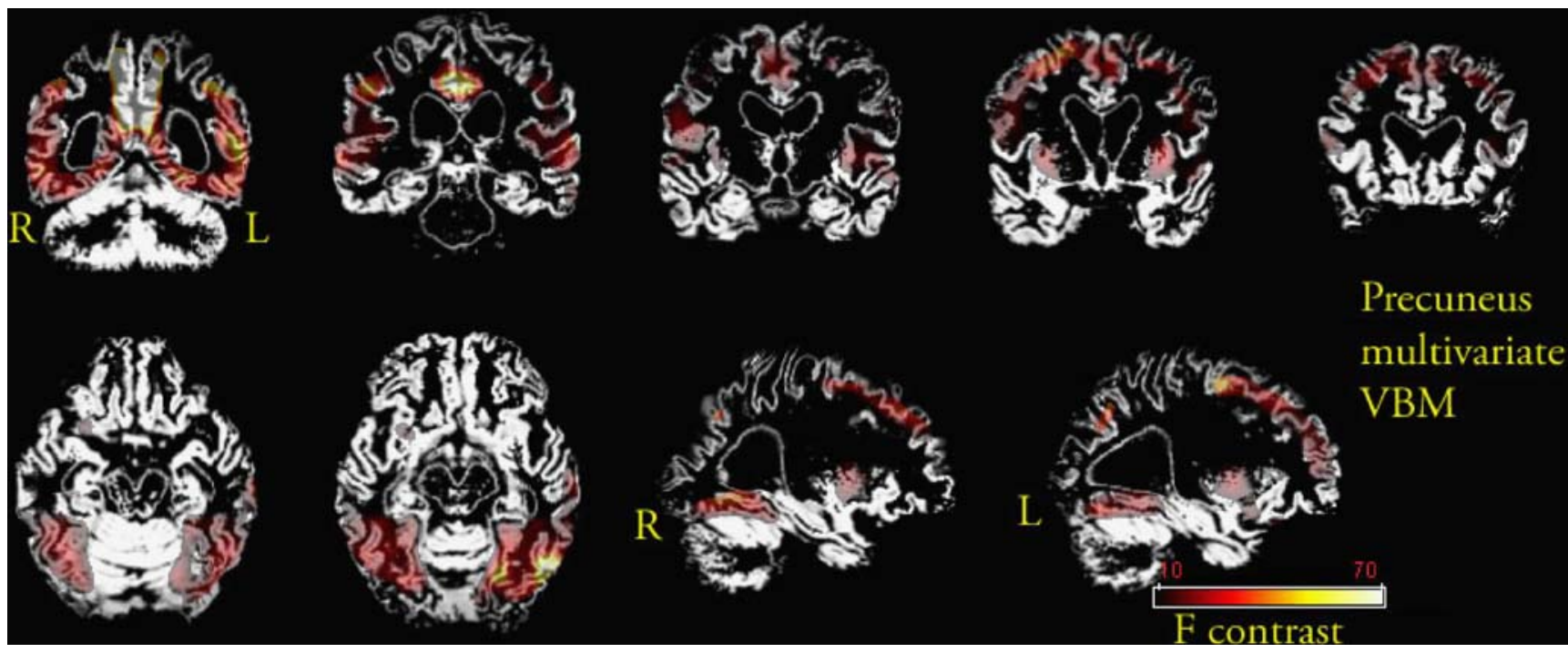

Fig. 5 Results of multivariate analysis with precuneus GM density as a single covariate. Since we were interested in showing whether there was accompanying hippocampal atrophy, we present unthresholded maps. By definition, precuneus atrophy was correlated with the location of the precuneus on the multivariate maps. Additionally, diffuse cortical atrophy was also associated with precuneus atrophy, especially in a biparietal cortical distribution. Interestingly, even on such high-sensitivity unthresholded statistical multivariate maps, there seems to be no association between precuneus atrophy and hippocampal atrophy, indicating two separate atrophy processes well below LAD patients on three out of four tests in this domain. As one would expect, learning capacity was impaired both in EAD and in LAD patients. However, EAD and LAD patients appeared to be equally affected for no statistically significant differences between these groups were found. Statistically significant group differences were also lacking in semantic memory and executive functioning. Semantic memory was affected in both patient groups and the apparent impairment in executive functioning could primarily be explained by a slower processing speed. Although visuospatial functioning was within the normal range for both patient groups, LAD patients had a better performance than EAD patients $(P=0.028)$.

Correlation analysis of precuneus volume with visuospatial functioning revealed a significant correlation (Pearson correlation coefficient 0.48 with $P=0.01$, one-tailed, since we expected atrophy of the precuneus to be associated only with lower visuospatial functioning scores). Due to the small

Table 2 Neuropsychological test scores

\begin{tabular}{|c|c|c|c|c|c|c|}
\hline \multirow[t]{2}{*}{ Test } & & \multicolumn{2}{|c|}{ EAD patients $(n=11)$} & \multicolumn{2}{|c|}{ LAD patients $(n=28)$} & \multirow{2}{*}{$\begin{array}{l}P- \\
\text { value }^{\mathrm{a}}\end{array}$} \\
\hline & & Mean & Standard error & Mean & Standard error & \\
\hline \multirow[t]{4}{*}{ Processing speed and attention } & $\begin{array}{l}\text { Wechsler adult intelligence scale } \\
\text { substitution } 1\end{array}$ & 15.83 & 4.86 & 31.32 & 2.32 & 0.021 \\
\hline & Trail making A2 & 172.68 & 36.88 & 71.31 & 17.88 & 0.034 \\
\hline & Digit span 1 - forward & 8.63 & 1.20 & 12.64 & 0.63 & 0.01 \\
\hline & Digit span - backward ${ }^{\mathrm{b}}$ & 5.30 & 1.07 & 7.59 & 0.57 & 0.085 \\
\hline \multirow[t]{2}{*}{ Episodic memory function } & Visual association test $3 \mathrm{~A}$ trial 1 & 0.57 & 0.67 & 1.37 & 0.37 & 0.328 \\
\hline & Visual association test $A$ trial $1+2$ & 1.51 & 1.32 & 3.83 & 0.7 & 0.095 \\
\hline \multirow[t]{3}{*}{ Semantic memory } & Fluency animals 4 & 10.78 & 1.63 & 10.96 & 0.94 & 0.926 \\
\hline & Fluency insects & 4.72 & 1.07 & 4.22 & 0.69 & 0.714 \\
\hline & $\begin{array}{l}\text { Wechsler adult intelligence scale } \\
\text { information } 1\end{array}$ & 11.07 & 2.39 & 9.98 & 1.16 & 0.711 \\
\hline \multirow[t]{2}{*}{ Executive functioning } & Trail making $\mathrm{B} 2^{\mathrm{c}}$ & 309.88 & 60.44 & 215.71 & 22.68 & 0.185 \\
\hline & Meander 5 & 2.19 & 0.55 & 3.06 & 0.33 & 0.213 \\
\hline Visuospatial functioning & Drawing task 6 & 9.95 & 0.68 & 11.87 & 0.41 & 0.028 \\
\hline
\end{tabular}

${ }^{\mathrm{a}}$ Univariate $\mathrm{F}$ tests for EAD and LAD patients corrected for gender and educational differences.

${ }^{\mathrm{b}}$ Corrected for forward span (\%).

${ }^{\mathrm{c}}$ Corrected for trail making A. 
sample size in our study we refrained from performing a data mining approach with all available neuropsychological data; we chose to test only for visuospatial functioning instead as stated in our hypothesis.

\section{Discussion}

We found that patients with EAD showed disproportionate and independent precuneus atrophy compared to patients with LAD. The specificity of precuneus atrophy is corroborated by its occurrence in the relative absence of significant hippocampal atrophy, despite worse MMSE scores. This is, to our knowledge, the first study attempting to dissociate atrophy of the precuneus from hippocampal atrophy by utilizing a multivariate approach. An analysis of available neuropsychological data did not show any difference between EAD and LAD in episodic memory function, semantic memory or executive functioning (Table 2), possibly reflecting no appreciable differences in hippocampal atrophy. However, we did find clear differences in visuospatial functioning and processing speed and attention.

Previous studies have also shown that the precuneus might have a smaller volume [3,4] or be functionally impaired $[5,6]$ in younger patients with AD. Additionally, precuneus volume has been found to be a good classifier in a group of patients with $\mathrm{AD}$ [30]. In most previous studies, the authors decided to set an arbitrary cut-off at 65 years of age and define younger patients as EAD and older patients as LAD. Our study confirmed the findings of the previous studies without imposing such an arbitrary cut-off. We chose to treat age as a continuous variable instead. Still, for communication purposes, we refer to the results as pertaining to 'younger' and 'older' AD patients despite the continuous nature of age, but it should be kept in mind that there might be overlap between the two groups and age alone might not be sufficient to characterize the two groups. A combination of precuneus volume and neuropsychological tests might offer a better classification, but we opted not to adopt this approach since that would have rendered our study cyclical (same data used to find classifying variables and then using these variables to classify the data into subgroups).

For the sake of completeness, we also performed a dichotomous analysis of our data with the cut-off at 65 years of age, which confirmed both the findings of the correlational analysis and the results of the previous VBM studies $[3,4]$. The severity of dementia, as measured by the MMSE, was higher in the younger patients although they appeared to have more preserved hippocampi. This finding is in agreement with those of other studies in EAD, in which the cognitive profile in EAD has been shown to be different from that in LAD [9], with visuospatial [11] and language disturbances [31] predominating.
From a neuropathological perspective, $\mathrm{AD}$ has been well described by the Braak and Braak staging [32, 33], with damage initiating in the entorhinal cortex, spreading to the medial temporal lobe and subsequently to the rest of the cortex. In fact, hippocampal neuronal cell loss is higher in older $\mathrm{AD}$ patients [34]. Only recently has it been suggested that younger $\mathrm{AD}$ patients might also have early synaptic loss in the PCC and precuneus [35] and that there might be precuneus volume loss in younger $\mathrm{AD}$ patients [36]. In a study that combined single photon emission CT (SPECT) with post-mortem Braak staging [37], hypometabolism of the precuneus was found in patients who were classified as belonging to either the Braak entorhinal or limbic stage (i.e. mild AD changes).

The precuneus and adjacent PCC are believed to play an important role in a default mode cortical network as they show the highest level of baseline metabolic activity in passive resting state studies [38]. Functionally these regions are involved in visuospatial processes such as the retrieval of the spatial context of events, detection of the speed of spatial targets and in processing egocentric aspects of movement [39-42]; for a detailed review we refer the reader to reference [8]. A study of stroke victims has shown that the precuneus is often associated with optic ataxia and that the precuneus might be the human equivalent of the 'parietal reach region' in the monkey [43]. In addition, the PCC and retrosplenial cortex appear to have a more general role in episodic memory functions whether spatial or nonspatial [44-46], and the anterior precuneus might be involved in retrieval mode and the posterior precuneus in retrieval success [8]. Consequently, it is not surprising to notice a different clinical profile in younger (rather than elderly) AD patients with a predominance of visuospatial disturbances [9-11]. A recent neuropsychological study comparing young and old $\mathrm{AD}$ patients has shown that young $\mathrm{AD}$ patients perform worse on language skills, visuospatial skills, executive functions and savings, while immediate and delayed memory impairment are equally affected in the two groups [47].

The strengths of our study include the wide age-range of the subjects with well-defined $\mathrm{AD}$, and the use of correlation-type VBM rather than a dichotomous comparison with an arbitrary cut-off. Additionally, our multivariate approach shows a clear dissociation between atrophy of the precuneus and the hippocampus. A potential limitation of our study is the absence of age-matched controls, which certainly has an effect on the relevance of our findings in older AD subjects. The disproportionate and independent atrophy found with younger age, however, is beyond doubt, as growth of the GM in this area in old AD subjects is extremely unlikely.

In summary, precuneus atrophy is a feature of EAD and might be noted even in the absence of hippocampal atrophy. Future studies might clarify the discriminating ability of precuneus atrophy in the presence or absence of hippocampal atrophy in a diagnostic setting. 
Acknowledgements G. Karas is the recipient of Grant 2001-014 by the "Stichting Alzheimer Nederland" and S.A.R.B. Rombouts is the recipient of Grant H00.17 from "Hersenstichting Nederland". The clinical database of the Alzheimer Center is supported by a grant from Stichting Dioraphte. Additional support was received from the Stichting Alzheimer \& Neuropsychiatrie Foundation.

Conflict of interest statement We declare that we have no conflict of interest.

\section{References}

1. Galton CJ, Patterson K, Xuereb JH et al (2000) Atypical and typical presentations of Alzheimer's disease: a clinical, neuropsychological, neuroimaging and pathological study of 13 cases. Brain 123: 484-498

2. Greicius MD, Geschwind MD, Miller BL (2002) Presenile dementia syndromes: an update on taxonomy and diagnosis. J Neurol Neurosurg Psychiatry 72:691-700

3. Ishii K, Kawachi T, Sasaki H et al (2005) Voxel-based morphometric comparison between early- and late-onset mild Alzheimer's disease and assessment of diagnostic performance of z score images. AJNR Am J Neuroradiol 26:333-340

4. Frisoni GB, Testa C, Sabattoli F et al (2005) Structural correlates of early and late onset Alzheimer's disease: voxel based morphometric study. J Neurol Neurosurg Psychiatry 76:112-114

5. Sakamoto S, Ishii K, Sasaki M et al (2002) Differences in cerebral metabolic impairment between early and late onset types of Alzheimer's disease. J Neurol Sci 200:27-32

6. Scarmeas N, Habeck C, Anderson KE et al (2004) Altered PET functional brain responses in cognitively intact elderly persons at risk for Alzheimer disease (carriers of the epsilon4 allele). Am J Geriatr Psychiatry 12:596-605

7. Yasuno F, Imamura T, Hirono N et al (1998) Age at onset and regional cerebral glucose metabolism in Alzheimer's disease. Dement Geriatr Cogn Disord 9:63-67

8. Cavanna AE, Trimble MR (2006) The precuneus: a review of its functional anatomy and behavioural correlates. Brain 129:564-583

9. Jacobs D, Sano M, Marder K et al (1994) Age at onset of Alzheimer's disease: relation to pattern of cognitive dysfunction and rate of decline. Neurology 44:1215-1220

10. Prvulovic D, Hubl D, Sack AT et al (2002) Functional imaging of visuospatial processing in Alzheimer's disease. Neuroimage 17:1403-1414

11. Fujimori M, Imamura T, Yamashita H et al (1998) Age at onset and visuocognitive disturbances in Alzheimer disease. Alzheimer Dis Assoc Disord 12:163-166

12. McKhann G, Drachman D, Folstein M et al (1984) Clinical diagnosis of Alzheimer's disease: report of the NINCDS-ADRDA Work Group under the auspices of Department of Health and Human Services Task Force on Alzheimer's Disease. Neurology 34:939-944

13. Qizilbash N (2002) Evidence-based dementia practice. Blackwell Science, Oxford, pp xxii, 893

14. Scheltens P, Leys D, Barkhof F et al (1992) Atrophy of medial temporal lobes on MRI in "probable" Alzheimer's disease and normal ageing: diagnostic value and neuropsychological correlates. J Neurol Neurosurg Psychiatry 55:967-972

15. Smith SM, Zhang Y, Jenkinson M et al (2002) Accurate, robust, and automated longitudinal and cross-sectional brain change analysis. Neuroimage 17:479-489

16. Chetelat G, Desgranges B, De La Sayette V et al (2002) Mapping gray matter loss with voxel-based morphometry in mild cognitive impairment. Neuroreport 13:1939-1943
17. Baron JC, Chetelat G, Desgranges B et al (2001) In vivo mapping of gray matter loss with voxel-based morphometry in mild Alzheimer's disease. Neuroimage 14:298-309

18. Ashburner J, Friston KJ (2000) Voxel-based morphometry - the methods. Neuroimage 11:805-821

19. Woods RP (2003) Characterizing volume and surface deformations in an atlas framework: theory, applications, and implementation. Neuroimage 18:769-788

20. Freeborough PA, Fox NC (1998) Modeling brain deformations in Alzheimer disease by fluid registration of serial 3D MR images. J Comput Assist Tomogr 22:838-843

21. Hellier P, Ashburner J, Corouge I et al (2002) Intersubject registration of functional and anatomical data using SPM. LNCS 2489:590-597

22. Davatzikos C, Genc A, Xu D et al (2001) Voxel-based morphometry using the RAVENS maps: methods and validation using simulated longitudinal atrophy. Neuroimage 14:1361-1369

23. Brett M, Anton JL, Valabregue R et al (2002) Region of interest analysis using an SPM toolbox. 8th International Conference on Functional Mapping of the Human Brain, Sendai, Japan

24. Wechsler D (1997) Wechsler adult intelligence scale - third edition. The Psychological Corporation, San Antonio

25. Reitan RM (1958) Validity of the trail making test as an indicator of organic brain damage. Percept Mot Skills 8:271-276

26. Lindeboom J, Schmand B, Tulner L et al (2002) Visual association test to detect early dementia of the Alzheimer type. J Neurol Neurosurg Psychiatry 73:126-133

27. Luteijn F, Van der Ploeg FAE (1983) Groninger intelligentie test. Swets and Zeitlinger, Lisse

28. Luria AR (1966) Higher cortical functions in man. Basic Books, New York

29. Beery KE, Buktenica NA (1989) Developmental test of visual-motor integration. Psychological Assessment Resources, Odessa, FL

30. Shiino A, Watanabe T, Maeda K et al (2006) Four subgroups of Alzheimer's disease based on patterns of atrophy using VBM and a unique pattern for early onset disease. Neuroimage 33:17-26

31. Imamura T, Takatsuki Y, Fujimori M et al (1998) Age at onset and language disturbances in Alzheimer's disease. Neuropsychologia 36:945-949

32. Braak E, Griffing K, Arai K et al (1999) Neuropathology of Alzheimer's disease: what is new since A. Alzheimer? Eur Arch Psychiatry Clin Neurosci 249(Suppl 3):14-22

33. Braak H, Braak E (1991) Neuropathological stageing of Alzheimerrelated changes. Acta Neuropathol (Berl) 82:239-259

34. Coleman PD, Flood DG (1987) Neuron numbers and dendritic extent in normal aging and Alzheimer's disease. Neurobiol Aging $8: 521-545$

35. Scheff SW, Price DA (2001) Alzheimer's disease-related synapse loss in the cingulate cortex. J Alzheimers Dis 3:495-505

36. Najlerahim A, Bowen DM (1988) Regional weight loss of the cerebral cortex and some subcortical nuclei in senile dementia of the Alzheimer type. Acta Neuropathol (Berl) 75:509-512

37. Bradley KM, O'Sullivan VT, Soper ND et al (2002) Cerebral perfusion SPET correlated with Braak pathological stage in Alzheimer's disease. Brain 125:1772-1781

38. Wang L, Zang Y, He Y et al (2006) Changes in hippocampal connectivity in the early stages of Alzheimer's disease: evidence from resting state fMRI. Neuroimage 31:496-504

39. Burgess N, Maguire EA, Spiers HJ et al (2001) A temporoparietal and prefrontal network for retrieving the spatial context of lifelike events. Neuroimage 14:439-453

40. Maguire EA, Burgess N, Donnett JG et al (1998) Knowing where and getting there: a human navigation network. Science 280:921-924

41. Maguire EA, Frackowiak RS, Frith CD (1997) Recalling routes around London: activation of the right hippocampus in taxi drivers. J Neurosci 17:7103-7110 
42. Mesulam MM, Nobre AC, Kim YH et al (2001) Heterogeneity of cingulate contributions to spatial attention. Neuroimage 13: $1065-1072$

43. Karnath HO, Perenin MT (2005) Cortical control of visually guided reaching: evidence from patients with optic ataxia. Cereb Cortex 15:1561-1569

44. Maddock RJ, Garrett AS, Buonocore MH (2001) Remembering familiar people: the posterior cingulate cortex and autobiographical memory retrieval. Neuroscience 104:667-676

45. Maguire EA (2001) The retrosplenial contribution to human navigation: a review of lesion and neuroimaging findings. Scand $\mathrm{J}$ Psychol 42:225-238
46. Shah NJ, Marshall JC, Zafiris O et al (2001) The neural correlates of person familiarity. A functional magnetic resonance imaging study with clinical implications. Brain 124:804-815

47. Bondi MW, Houston WS, Salmon DP et al (2003) Neuropsychological deficits associated with Alzheimer's disease in the very-old: discrepancies in raw vs. standardized scores. J Int Neuropsychol Soc 9:783-795

48. Mai JK, Assheuer J, Paxinos G (2004) Atlas of the human brain. Academic Press, San Diego, p 246

49. Chau W, McIntosh AR (2005) The Talairach coordinate of a point in the MNI space: how to interpret it. Neuroimage 25: $408-416$ 